\title{
Patellar tendon buckling is altered with age
}

\author{
Laura Chernak Slane ${ }^{\mathrm{a}, *}$, Félix Dandois ${ }^{\mathrm{a}}$, Stijn Bogaerts ${ }^{\mathrm{a}, \mathrm{b}}$, Hilde Vandenneucker ${ }^{\mathrm{a}, \mathrm{b}, 1}$, \\ Lennart Scheys ${ }^{\mathrm{a}, \mathrm{b}, 1}$
}

a Institute for Orthopaedic Research and Training (IORT), KU Leuven, UZ Pellenberg, Weligerveld 1/Blok 1, Pellenberg, 3212 Leuven, Belgium

${ }^{\mathrm{b}}$ University Hospitals Leuven, Campus Pellenberg, Pellenberg, Belgium

\section{A R T I C L E I N F O}

Article history:

Received 22 January 2018

Revised 22 March 2018

Accepted 30 April 2018

\section{Keywords:}

Crimp

Rupture

Tendinopathy

Sex-based differences

\begin{abstract}
A B S T R A C T
Recent evidence has revealed that the patellar tendon exhibits buckling during passive knee extension, wherein the tendon folds back onto itself. The clinical relevance of such buckling is unclear, but it has been suggested that it serves to protect the patellar tendon from rupture when subjected to a sudden extreme contraction. Although prior evidence suggests buckling occurs universally, it is poorly understood, and may be influenced by age and sex. Healthy adults ( $n=41$, aged 21-80 years) were recruited to assess age- and sex-based differences in patellar tendon buckling during passive knee extension. 93\% of subjects exhibited buckling in extension, with buckling more prominent in the distal tendon. No ageor sex-based differences in buckling magnitude were observed, but a significant age-based difference in buckling angle was found, with the tendon unbuckling later in flexion in younger adults compared with middle-aged $(p=0.025)$ and older $(p=0.014)$ adults. Intrinsic factors were also linked with buckling; for example, smaller maximum knee extension (i.e. less flexibility) correlated with smaller buckling magnitude ( $p=0.037, R^{2}=0.116$ ), suggesting a link between patellar tendon buckling and joint-level mechanics. These results suggest that buckling is an inherent component of normal knee function that older adults may be failing to take advantage of, predisposing them to injury. Further study will be critical to elucidate the clinical implications of patellar tendon buckling.
\end{abstract}

(C) 2018 IPEM. Published by Elsevier Ltd. All rights reserved.

\section{Introduction}

Tendon injuries remain poorly understood, making them difficult to both prevent and treat. Some risk factors, like age and sex, have been identified, but these factors seem to affect different tendons disparately. For example, although the Achilles and patellar tendons have similar functional behavior, the influence of age and sex on injury incidence varies; whereas Achilles tendon rupture and tendinopathy occur most commonly in middle-age [1,2], ruptures in the patellar tendon occur much later in life [1], and no clear link has been found between age and patellar tendinopathy [3-6]. Likewise, though males are at higher risk for Achilles and patellar ruptures $[1,7,8]$, as well as patellar tendinopathy $[5,9,10]$, Achilles tendinopathy is equally common in males and females [2]. It could be postulated that the differences arise due to their different anatomy; notably the subtendons of the Achilles [11], and the presence of the patella bone embedded within the patellar tendon, but histological data support the categorization of the patellar tendon as more similar in composition to tendon rather than ligament

\footnotetext{
* Corresponding author.

E-mail address: Laura.C.Slane@gmail.com (L.C. Slane).

1 Equal contributions.
}

[12]. Alternatively, the various hypotheses that have been proposed to explain the links between tendon injuries, age and sex, often focus on age- or sex-based changes in tendon tissue, which thus fail to explain why the Achilles and patellar tendons would be affected differently by these factors, thereby suggesting that factors other than broad tissue-based changes must play a role in injury development.

Joint-level mechanics may be key to understanding the difference in Achilles and patellar tendon injury incidence. One factor that has yet to be fully considered is the buckling of the patellar tendon that occurs when the knee is relaxed and in an extended posture [13]. This tendon buckling, in which the tendon folds back on itself, has rarely been described in the literature and is not widely recognized as a characteristic of normal knee function. In fact, the few studies that described buckling previously have theorized that it is a sign of tendon damage [14-16], a theory called into question by our previous observation of universal buckling in the patellar tendons of healthy young adults ( $n=20$ [17]). Although the clinical relevance of buckling has yet to be elucidated, observations of region-specific differences may provide a clue. Specifically, it was found that buckling occurred during a greater portion of the flexion cycle in the less-frequently injured distal tendon [18-20], suggesting that buckling may serve to protect this region of the 
Table 1

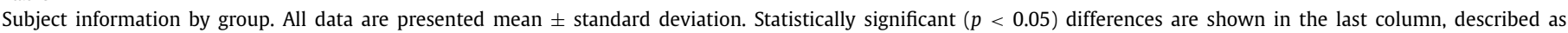
either between young and middle-aged adults (YM), young and older adults (YO), middle-aged and older adults (MO), or no significant differences between groups (n.s.).

\begin{tabular}{|c|c|c|c|c|}
\hline & Young $(21-40)$ & Middle-aged (41-60) & Older $(61+)$ & \\
\hline Sex & $10 \mathrm{M}, 10 \mathrm{~F}$ & $5 \mathrm{M}, 5 \mathrm{~F}$ & $6 \mathrm{M}, 5 \mathrm{~F}$ & \\
\hline Age (yrs) & $28 \pm 4$ & $51 \pm 4$ & $71 \pm 6$ & $Y M, Y O, M O$ \\
\hline Height $(\mathrm{cm})$ & $174 \pm 7$ & $175 \pm 13$ & $166 \pm 8$ & n.s. \\
\hline Weight (kg) & $70 \pm 11$ & $75 \pm 14$ & $71 \pm 12$ & n.s. \\
\hline BMI & $23 \pm 3$ & $24 \pm 3$ & $26 \pm 2$ & YO \\
\hline Max. extension (deg flexion) & $6 \pm 7$ & $4 \pm 3$ & $5 \pm 4$ & n.s. \\
\hline VISA-P score & $98 \pm 3$ & $99 \pm 2$ & $92 \pm 6$ & YO, MO \\
\hline
\end{tabular}

tendon from injury. A link between buckling, age and sex, could help to explain why the injury rate of the Achilles and patellar tendons are seemingly affected differently by age and sex differences. In addition, prior observations found that the magnitude of buckling was increased in subjects who were less active and older, suggesting that excessive buckling could be a sign of tissue degeneration, though all subjects in the prior study were relatively healthy and from a narrow age range (23-37). Thus, we undertook this follow-up study to evaluate whether there are any ageor sex- based differences in patellar tendon buckling. We hypothesized that (1) buckling magnitude would increase with age, (2) buckling magnitude would be greater in males, and (3) buckling angles would be smaller in older adults who are more predisposed to injury.

\section{Methods}

In compliance with the Commission for Medical Ethics UZ KU Leuven (protocol \#s59014), healthy subjects (aged 40+) were recruited and provided written consent. Subjects were included only if they had no self-reported history of knee injury, surgery or musculoskeletal disorder $(n=21)$. Upon arrival, all subjects were asked to report their height and weight, and to complete the VISA-P questionnaire [21] to assess their patellar tendon health (Table 1).

Subjects were seated in an isokinetic testing device (Biodex Medical Systems, Shirley, NY, USA) and a twin-axis electric goniometer (Biometrics Ltd, Newport, UK) was used to record knee flexion angle. The session began with a six-minute warm-up period through the subjects' range of motion (ROM) from maximum extension to $90^{\circ}$ of flexion, to precondition the muscle-tendon tissues [22]. Next, an ultrasound transducer (10 MHz, L14-5/38, Ultrasonix Corporation, Richmond, BC) was positioned over the patellar tendon to collect dynamic (70 fps) radiofrequency (RF) data. Because the transducer's width $(38 \mathrm{~mm}$ ) was insufficient to capture the full length of the patellar tendon, data were collected in a random order from either the proximal or distal tendon. For both locations, anatomical landmarks were identified (either the distal edge of the patella or the notch of the tibial tuberosity) and kept in view. Five repeat trials of passive flexion/extension through the ROM were collected for each transducer position. Next, a hypoechoic wire was secured over the midportion of the tendon, and flexion/extension cycles were repeated, with three trials collected from each transducer position. In post-hoc analyses, the shadow created by the wire was used to compute the full tendon length. LabVIEW (National Instruments Corporation, Austin, TX, USA) was used during all trials to synchronize ultrasound data with the electric goniometer.

Ultrasound B-mode images were reconstructed and evaluated in MATLAB (R2015B, Mathworks, Natick, MA, USA). Images were viewed sequentially, from knee extension to flexion, and the frame at which the tendon borders became continuous (i.e. the tendon is fully unbuckled) was identified visually, with the corresponding knee angle extracted and termed the buckling angle (Fig. 1). This analysis was repeated three times per trial, with the results aver- aged to determine a mean distal and mean proximal buckling angle for each subject, if present. Poor quality trials were removed, such that on average $9 \pm 1$ trials (of 10 total) were included in the analysis per subject. The distance between tendon insertion points was estimated in MATLAB from the images collected with the hypoechoic wire in place and was computed as the sum of the distance between the distal edge of the patella and the wire, and the wire and the tibial tuberosity. Because the tendon buckles in extension, this metric is not equivalent to the tendon length. Distance measures were repeated three times for each image, with the average taken for each subject. From this measurement, buckling magnitude could be computed. Buckling magnitude is a measure of how much of the tendon's length is buckled in maximum extension, and is computed mathematically as the difference in the distance between insertion points at the maximum buckling angle (i.e. proximal or distal) and maximum extension, normalized to the tendon length at $90^{\circ}$ [17].

To enable broader assessments of any potential age-related changes, previously reported data [17] from healthy young adults (aged $<40, n=20$ ) collected using the same setup were also included in the analysis. Thus, in total, data from 41 adults were assessed. For group-wise comparisons, subjects were divided by age: young (aged 21-40, $n=20$ ), middle-aged (41-60, $n=10$ ) and older $(61+, n=11)$ adults. ANOVAs were used to assess group-wise differences in intrinsic characteristics (age, BMI, maximum extension, maximum tendon length, VISA-P Score) and buckling results (distal and proximal buckling angle, buckling magnitude). Significant interactions were followed up with Tukey post-hoc tests $(p<0.05)$. $t$-Tests were used to assess sex-based differences in terms of the intrinsic characteristics and buckling results described above. Linear regressions were then used to test for correlations between BMI, maximum extension, maximum tendon length, and VISA-P score and buckling results. Linear regressions also tested for a link between age and maximum extension. Finally, the intrinsic characteristics of subjects with and without evidence of distal and proximal buckling were compared using $t$-tests. All statistics were performed in SPSS (IBM Corp., Armonk, NY, USA), with $p<0.05$ taken as significant.

\section{Results}

Buckling was observed in 38 of the 41 subjects (Figure 1). Of these 38, all exhibited buckling in the distal tendon, and 26 also exhibited proximal buckling. No statistical differences were found between subjects with and without buckling in terms of age, BMI, maximum extension, maximum tendon length or VISA-P score.

In terms of group-wise comparisons, an aging effect was observed (Fig. 2), with young adults exhibiting a significantly larger buckling angle (i.e. unbuckling occurred at a more flexed posture) compared with middle-aged $(p=0.025)$ and older adults $(p=0.014)$. No group-wise differences in terms of proximal buckling or buckling magnitude were found. There were some intrinsic differences between age groups; young subjects had significantly lower BMIs than older subjects $(p=0.04)$, and older subjects 

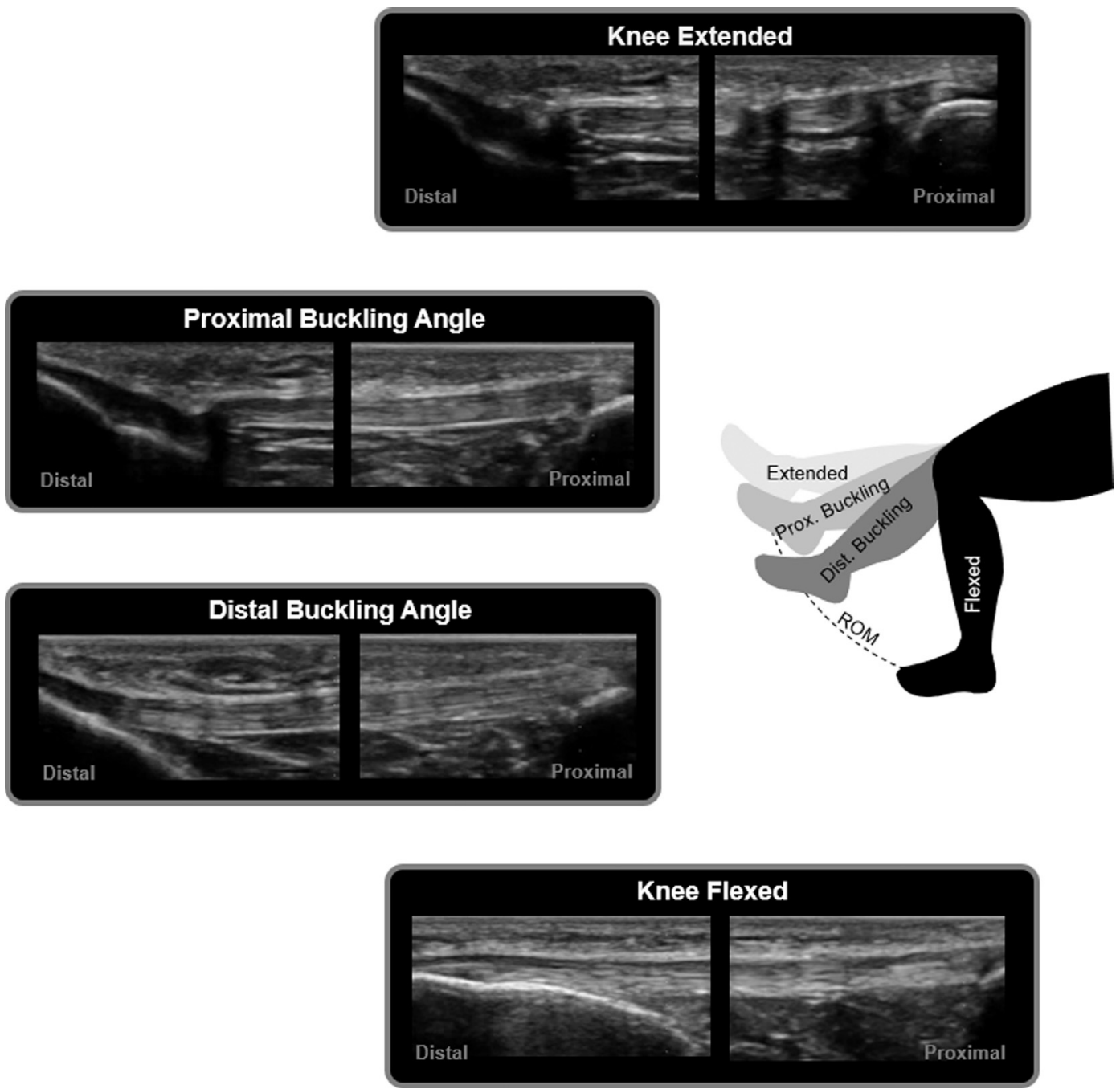

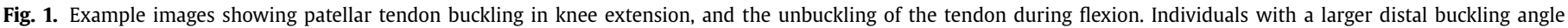
spend more of their range of motion unbuckling rather than lengthening.

scored significantly worse $(p<0.001)$ on the VISA-P than both young and middle-aged subjects.

No differences between males and females in terms of distal or proximal buckling angle, or buckling magnitude were observed. There were some intrinsic differences between groups however, with female subjects being able to reach a significantly more extended posture $(p=0.004)$, and having significantly shorter tendons $(p=0.004)$.

Some general links between buckling results and intrinsic factors were also observed. Smaller maximum knee extension (i.e. less flexibility) was correlated with a more flexed distal $(p<0.001$, $\left.R^{2}=0.299\right)$ and proximal $\left(p<0.001, R^{2}=0.601\right)$ buckling angle, and a smaller buckling magnitude $\left(p=0.037, R^{2}=0.116\right)$. Buckling magnitude was also smaller in individuals with longer tendons $\left(p=0.009, R^{2}=0.176\right)$. BMI and VISA-P score had no effect on buckling results. In the young adult group, there was a link between age and ROM $\left(p=0.04, R^{2}=0.2\right)$, but no link was found when all subjects were grouped together $\left(p=0.2, R^{2}=0.04\right)$.

\section{Discussion}

In contrast to our first two hypotheses, we observed no effect of age or sex on buckling magnitude. Also, in contrast with our third hypothesis, we found no age-based effect on proximal buckling angle, and that distal buckling angles were significantly smaller in the middle-aged and older populations compared with young adults. We had hypothesized that buckling would increase with age due to prior observations of a link between extreme buckling and tissue damage [14-16]. The results of this study suggest that age-based changes in buckling may arise from an entirely different mechanism than pathological changes in buckling, and may be a precursor for injury rather than a symptom of preclinical injury. Smaller buckling angles in the middle-aged and older adults suggest that the distal tendon becomes taut (i.e. unbuckled) at a smaller flexion angle. This result means that over the same range of motion, the patellar tendons of young adults are unbuckling over a greater proportion of the flexion cycle. In contrast, the patellar tendons of older adults are fully unbuckled and taut earlier in flexion, such that the remainder of the overall lengthening required for flexion must be accomplished by lengthening of the muscle-tendon unit (MTU). Such lengthening could occur in any of the contributing structures; the patellar tendon, quadriceps tendon or quadriceps muscle. For example, if one considers the patellar tendon as the source for lengthening, this would imply that the patellar tendons of older adults undergo more strain during passive flexion. Mechanically, increased strain could be due to changes in the material properties of older tendons, or changes in stress through the tendon. In the first case, if young and old adult tendons were exposed to the same stress during passive knee flexion, then an increase in strain would mean that older adult tendons have a re- 


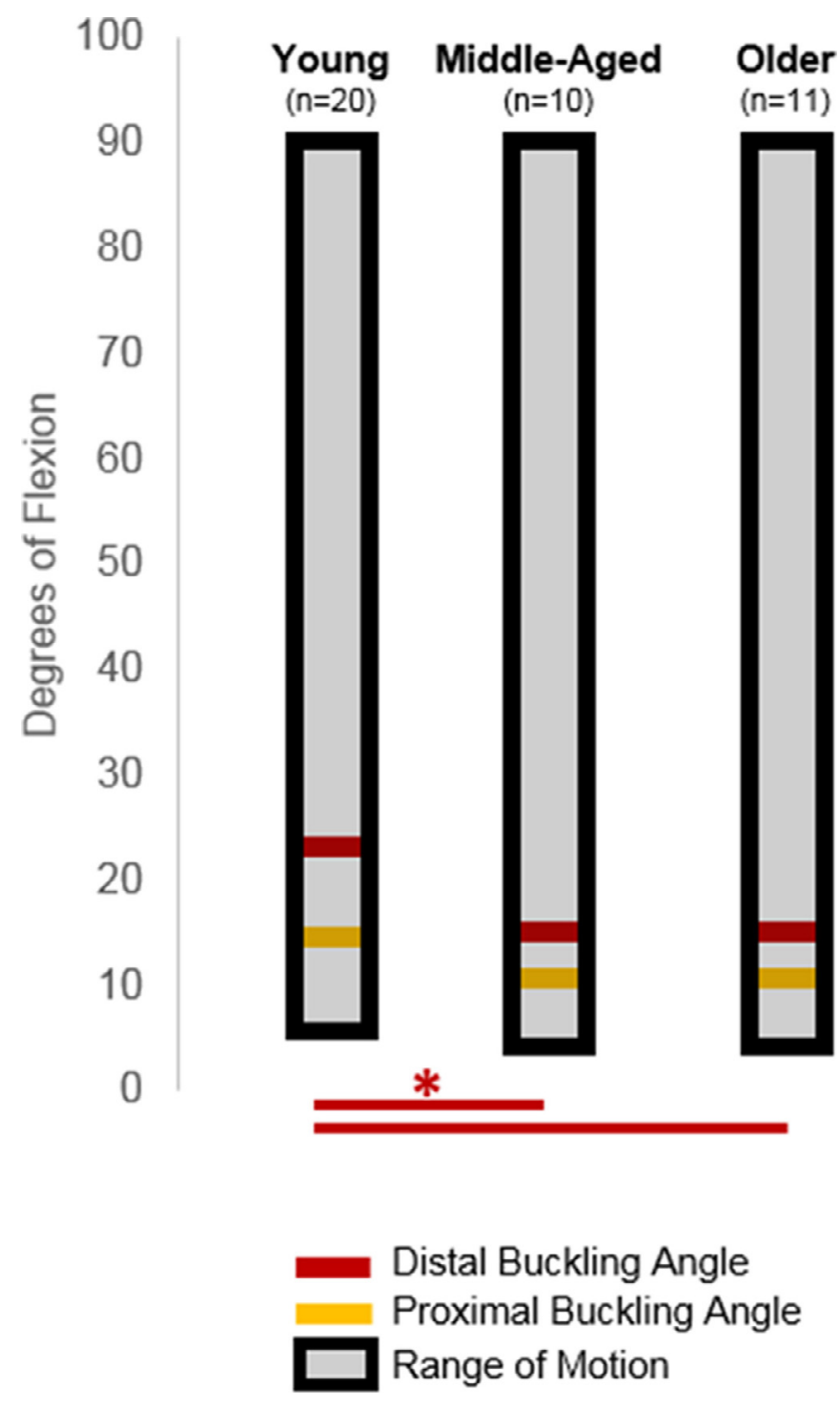

Fig. 2. Range of motion and proximal buckling angle were similar between all age groups, but young adults had a significantly larger distal buckling angle than middle-aged $(p=0.025)$ and older $(p=0.014)$ adults. Thus, young adults were taking advantage of patellar tendon unbuckling over a larger percent of the flexion cycle.

duced elastic modulus. Reduced elastic moduli with age have been previously observed in the in vivo Achilles [23,24], though the results may be confounded by differences in the force-generating capacity of older adults; recent in vivo studies comparing adults of the same strength (Achilles tendon, [25]) or at the same force (patellar tendon, [26]), found that any apparent age-related differences in elastic modulus were reduced to negligible. Ex-vivo studies have also produced conflicting results regarding the effects of age on tendon elastic modulus [27-29], such that more work is clearly needed to clarify this possible aging effect. On the other hand, increased strain in the patellar tendons of older adults could arise in tendons with the same material properties if subjected to larger stresses during passive knee flexion. Increased stress could arise due to either increased force through the patellar tendon or a reduction in tendon cross-sectional area. Cross-sectional area reduction seems unlikely; most studies suggest that either age does not influence cross-sectional area (patellar tendon [30]), or leads to increases in cross-sectional area (Achilles tendon [24,25,31]). Likewise, age-based variations in other tissues of the MTU (quadriceps tendon or muscle), or changes in parameters such as load shar- ing across the knee, moment arm length, etc., could also influence length changes, such that further study is warranted to understand the meaning of the results presented here. Musculoskeletal models may be of use in further investigating the possible causes of these age-based differences, as intuiting the complex interactions of muscle-tendon tissues can lead to incorrect assumptions [32].

Buckling of the patellar tendon may be clinically relevant, though the specific function has yet to be elucidated. One hypothesis is that buckling may serve a protective role, similar to microstructural fiber crimping, which is considered to serve to protect tendon from high strains and serve as a 'safety mechanism' against sudden forces [33]. Likewise, non-traumatic acute patellar tendon ruptures are thought to be caused by sudden extreme quadriceps contractions [34]. In such situations, buckling may actually protect the tendon from a high impulse load causing high strains. This hypothesis would be consistent with the finding here that the patellar tendons of young adults, who are at less risk for tendon rupture [1], show greater buckling. Intriguingly, micro-structural crimp has also been observed to reduce with age $[33,35]$, which likewise has been hypothesized to put older tendons at higher risk of injury. Additional testing of subjects at high risk for patellar tendon rupture (e.g. older males), or contralateral comparisons post-injury, might shed light on this potential clinical link.

An interesting observation in this study was the presence of regional variations in patellar tendon buckling. Buckling occurred more commonly in the distal tendon, with age-based changes only observed distally. These observations contribute to the growing body of research that tendon tissue is heterogeneous with regional variations. Such observations may be relevant in the context of injury, which also varies regionally, with the proximal tendon more at risk [18-20]. One possible mechanism is that buckling is shielding the distal tendon from external compression, which in turn is protecting it from developing tendinopathy [36].

In contrast to our prior study of adults under 40 , we did not find a link between age and buckling magnitude. On closer analysis of the young adult group, we found a significant correlation $\left(p=0.04, R^{2}=0.2\right)$ between age and maximum extension. Given the significant correlation between maximum extension and buckling magnitude $(p<0.001)$, the age-based link observed previously may have been confounded by the link between age and maximum knee extension. In this larger dataset $(n=41)$, we found no link between maximum extension and age $\left(p=0.2, R^{2}=0.04\right)$, which likely explains the lack of correlation between age and buckling magnitude in this larger dataset, and reiterates the need for larger datasets in future studies.

Although buckling was observed in most subjects, three subjects showed no evidence of buckling. No statistical difference was found between subjects with and without buckling in terms of any intrinsic factors, so it is unclear why these individuals did not exhibit buckling. During testing, we observed that some subjects found it challenging to relax during movement trials, inadvertently contracting their muscles during motion. This may be the reason why some individuals did not exhibit buckling, as it has been observed to be present only when the quadriceps are uncontracted [13]. In our prior study, we found it relatively straightforward to observe muscle activation during trials, such that EMG data were deemed noncritical for this study. However, in retrospect, without EMG confirmation, a failure of these subjects to relax cannot be ruled out as the reason for a lack of buckling, such that EMG observation may be critical, especially in older adult populations. It is also possible that some individuals do not exhibit buckling of the patellar tendon at all. The small sample size $(n=41)$ and observed differences between groups in terms of BMI and VISA-P scores are both limitations of this study. Additional work is necessary to better elucidate the universality of patellar tendon buckling. 
Another limitation of this study was that we previously found reduced buckling magnitude in subjects who were more physically active [37]. Unfortunately, although we administered the same survey as was used previously, with given examples of mild, moderate and strenuous activities, we observed a notable difference between how individuals of different ages completed this form, so we choose not include these data in the study. However, this meant that we could not follow up on our previous observation that buckling magnitude was linked with reduced regular activity, which would be of interest in future studies. An additional inherent limitation is that using a $2 \mathrm{D}$ imaging modality to evaluate a 3D phenomenon is inherently limited, as with other ultrasoundbased studies; future studies of buckling using 3D ultrasound may shed light on how buckling arises in three dimensions. Finally, our study was motivated in part by observations of differing injury incidence rates in the Achilles and patellar tendon which broad tissue-level differences due to age or sex could not explain. In this study, we investigated the possibility that joint-level changes in patellar tendon buckling could be a contributor to injury incidence rates in the patellar tendon. However, there are other factors that may contribute to the differences in injury incidence rates between the Achilles and patellar tendons. For example, the anatomies of the Achilles and patellar tendon differ substantially; the Achilles is characterized by subtendons that arise in the muscles of the triceps surae [38], whereas the patellar tendon has the intratendinous sesamoid patella bone. Secondly, a recent study suggested that tendinopathy may have opposite effects on the material properties of these two tendons [39]. Clearly more work is necessary to better discriminate between these and other potential contributing factors (e.g. microstructural changes).

\section{Conclusions}

Patellar tendon buckling was observed in $93 \%$ of subjects, with buckling more prominent in the distal tendon. In contrast to our hypotheses, no sex-based differences in terms of buckling angle or buckling magnitude were observed, and no links between age and buckling magnitude were found. However, distal buckling was more prominent in younger subjects compared with middle-aged and older subjects. This buckling may be clinically relevant; patellar tendon rupture is thought to occur following a sudden extreme contraction of the quadriceps. Young adults with more buckling, and thus more slack in their tendons, may be better able to handle such contractions and thereby avoid rupture. This hypothesis is consistent with the fact that young adults are less likely to rupture their patellar tendons. Further study is necessary to elucidate the clinical implications of patellar tendon buckling.

\section{Acknowledgments}

The authors gratefully acknowledge the assistance of Lore Hermans and Kristel Van de Loock. This work was supported by the European Knee Society and the National Institutes of Health [F32 AR069459-01].

\section{Competing interests}

The authors have no competing interests to declare.

\section{Supplementary materials}

Supplementary material associated with this article can be found, in the online version, at doi:10.1016/j.medengphy.2018.04. 024.

\section{References}

[1] Clayton RAE, Court-Brown CM. The epidemiology of musculoskeletal tendinous and ligamentous injuries. Injury 2008;39:1338-44. doi:10.1016/j.injury.2008. 06.021

[2] de Jonge S, van den Berg C, de Vos RJ, van der Heide HJL, Weir A, Verhaar JAN, et al. Incidence of midportion Achilles tendinopathy in the general population. Br J Sports Med 2011;45:1026-8. doi:10.1136/bjsports-2011-090342.

[3] Ferretti A. Epidemiology of jumper's knee. Sport Med 1986;3:289-95. doi:10. 2165/00007256-198603040-00005.

[4] Gaida JE. Are unilateral and bilateral patellar tendinopathy distinguished by differences in anthropometry, body composition, or muscle strength in elite female basketball players? Br J Sports Med 2004;38:581-5. doi:10.1136/bjsm. 2003.006015

[5] Lian ØB, Engebretsen L, Bahr R. Prevalence of jumper's knee among elite athletes from different sports. Am J Sports Med 2005;33:561-7. doi:10.1177| 0363546504270454.

[6] Witvrouw E, Bellemans J, Lysens R, Danneels L, Cambier D. Intrinsic risk factors for the development of patellar tendinitis in an athletic population. Am J Sports Med 2011;29:190-5. doi:10.1177/03635465010290021201.

[7] Jozsa L, Kvist M, Balint BJ, Reffy A, Jarvinen M, Lehto M, et al. The role of recreational sport activity in Achilles tendon rupture. Am J Sports Med 1989;17:338-43. doi:10.1177/036354658901700305.

[8] Negrin LL, Nemecek E, Hajdu S. Extensor mechanism ruptures of the knee: differences in demographic data and long-term outcome after surgical treatment. Injury 2015;46:1957-63. doi:10.1016/j.injury.2015.06.042.

[9] de Vries AJ, van der Worp H, Diercks RL, van den Akker-Scheek I, Zwerver J. Risk factors for patellar tendinopathy in volleyball and basketball players: a survey-based prospective cohort study. Scand J Med Sci Sports 2015;25:67884. doi:10.1111/sms.12294.

[10] Zwerver J, Bredeweg SW, van den Akker-Scheek I. Prevalence of jumper's knee among nonelite athletes from different sports. Am J Sports Med 2011;39:19848. doi: $10.1177 / 0363546511413370$.

[11] Handsfield GG, Slane LC, Screen HRC. Nomenclature of the tendon hierarchy: an overview of inconsistent terminology and a proposed size-based naming scheme with terminology for multi-muscle tendons. J Biomech 2016;49. doi:10.1016/j.jbiomech.2016.06.028.

[12] Rumian AP, Wallace AL, Birch HL. Tendons and ligaments are anatomically distinct but overlap in molecular and morphological features - a comparative study in an ovine model. J Orthop Res 2007:25:458-64. doi:10.1002/jor:20218.

[13] Slane LC, Bogaerts S, Mihejeva I, Scheys L. Evidence of patellar tendon buckling during passive knee extension. Knee 2016;23. doi:10.1016/j.knee.2016.06.005.

[14] Schweitzer ME, Mitchell DG, Ehrlich SM. The patellar tendon: thickening, internal signal buckling, and other MR variants. Skelet Radiol 1993;22:411-16.

[15] Berlin R, Levinsohn EM, Chrisman H. The wrinkled patellar tendon: an indication of abnormality in the extensor mechanism of the knee. Skelet Radiol 1991;20. doi:10.1007/BF00241662.

[16] Reiff D, Heenan S, Heron C. MRI appearances of the asymptomatic patellar tendon on gradient echo imaging. Skelet Radiol 1995;24. doi:10.1007/ BF00198074.

[17] Slane LC, Bogaerts S, Mihejeva I, Scheys L. Evidence of patellar tendon buckling during passive knee extension. Knee 2016:6-11. doi:10.1016/j.knee.2016. 06.005

[18] Cook JL, Khan KM, Harcourt PR, Kiss ZS, Fehrmann MW, Griffiths LR, et al. Patellar tendon ultrasonography in asymptomatic active athletes reveals hypoechoic regions: a study of 320 tendons. Inst Heal Biomed Innov 1998;8(2):73-7

[19] Johnson DP, Wakeley CJ, Watt I. Magnetic resonance imaging of patellar tendonitis. J Bone Jt Surg Br 1996;78-B:452-7.

[20] Shalaby M, Almekinders LC. Patellar tendinitis: the significance of magnetic resonance imaging findings. Am J Sport Med 1999;27:345-9.

[21] Visentini PJ, Khan KM, Cook JL, Kiss ZS, Harcourt PR, Wark JD. The VISA score: an index of severity of symptoms in patients with jumper's knee (patellar tendinosis). Victorian Institute of Sport Tendon Study Group. J Sci Med Sport 1998;1:22-8.

[22] Hawkins D, Lum C, Gaydos D, Dunning R. Dynamic creep and pre-conditioning of the Achilles tendon in-vivo. J Biomech 2009;42:2813-17. doi:10.1016/j. jbiomech.2009.08.023.

[23] Onambele GL, Narici M V, Maganaris CN. Calf muscle-tendon properties and postural balance in old age. J Appl Physiol 2006;100:2048-56. doi:10.1152/ japplphysiol.01442.2005.

[24] Stenroth L, Cronin NJ, Peltonen J, Korhonen MT, Sipilä S, Finni T. Triceps surae muscle-tendon properties in older endurance- and sprint-trained athletes. J Appl Physiol 2015;2015 jap00511. doi:10.1152/japplphysiol.00511.2015.

[25] Stenroth L, Peltonen J, Cronin NJ, Sipilä S, Finni T. Age-related differences in Achilles tendon properties and triceps surae muscle architecture in vivo. J Appl Physiol 2012;113 1537-44. doi:10.1152/japplphysiol.00782.2012.

[26] Couppé C, Hansen P, Kongsgaard M, Kovanen V, Suetta C, Aagaard P, et al. Mechanical properties and collagen cross-linking of the patellar tendon in old and young men. J Appl Physiol 2009;107 880-6. doi:10.1152/japplphysiol.00291. 2009.

[27] Thorpe CT, Udeze CP, Birch HL, Clegg PD, Screen HRC. Capacity for sliding between tendon fascicles decreases with ageing in injury prone equine tendons: a possible mechanism for age-related tendinopathy? Eur Cell Mater 2013;25:48-60 
[28] Vogel H. Influence of maturation and aging on mechanical and biochemical properties of connective tissue in rats. Mech Ageing Dev 1980;14 283-92. doi:10.1016/0047-6374(80)90002-0.

[29] Blevins FT, Hecker AT, Bigler GT, Boland AL, Hayes WC. The effects of donor age and strain rate on the biomechanical properties of bonepatellar tendon-bone allografts. Am J Sports Med 1994;22:328-33. doi:10.1177/ 036354659402200306.

[30] Couppé C, Suetta C, Kongsgaard M, Justesen L, Hvid LG, Aagaard P, et al. The effects of immobilization on the mechanical properties of the patellar tendon in younger and older men. Clin Biomech 2012;27:949-54. doi:10.1016/j. clinbiomech.2012.06.003.

[31] Magnusson SP, Beyer N, Abrahamsen H, Aagaard P, Neergaard K, Kjaer M. Increased cross-sectional area and reduced tensile stress of the Achilles tendon in elderly compared with young women. J Gerontol A Biol Sci Med Sci 2003;58:123-7

[32] Epstein M, Wong M, Herzog W. Should tendon and aponeurosis be considered in series? J Biomech 2006;39:2020-5. doi:10.1016/j.jbiomech.2005.06.011.

[33] Patterson-Kane J, Firth E, Goodship A, Parry D. Age-related differences in collagen crimp patterns in the superficial digital flexor tendon core region of untrained horses. Aust Vet J 1997;75:39-44. doi:10.1111/j.1751-0813.1997. tb13829.x.
[34] Saragaglia D, Pison A, Rubens-Duval B. Acute and old ruptures of the extensor apparatus of the knee in adults (excluding knee replacement). Orthop Traumatol Surg Res 2013;99:S67-76. doi:10.1016/j.otsr.2012.12.002.

[35] Wilmink J, Wilson AM, Goodship AE. Functional significance of the morphology and micromechanics of collagen fibres in relation to partial rupture of the superficial digital flexor tendon in racehorses. Res Vet Sci 1992;53:354-9. doi:10.1016/0034-5288(92)90139-S.

[36] Cook JL, Purdam C. Is compressive load a factor in the development of tendinopathy. Br J Sports Med 2012;46 163-8. doi:10.1136 bjsports-2011-090414.

[37] Slane LC, Bogaerts S, Mihejeva I, Scheys L. Evidence of patellar tendon buckling during passive knee extension. Knee 2016;23(5):801-6.

[38] Handsfield GG, Inouye JM, Slane LC, Thelen DG, Miller GW, Blemker SS. A 3D model of the Achilles tendon to determine the mechanisms underlying nonuniform tendon displacements. J Biomech 2017;51. doi:10.1016/j.jbiomech. 2016.11.062.

[39] Coombes BK, Tucker K, Vicenzino B, Vuvan V, Mellor R, Heales L et al. Achilles and patellar tendinopathy display opposite changes in elastic properties: a shear wave elastography study. Scand J Med Sci Sport 2017. doi:10.1111/sms. 12986. 